\title{
Direct searches for Dark Matter
}

\section{Elena Aprile*}

Physics Department, Columbia University, 538 W 120 St New York , NY 10027

E-mail: agedastro.columbia.edu

\begin{abstract}
An increasing number of astronomical and astrophysical observations strongly suggest that the Universe is composed of more than $96 \%$ of invisible matter and energy and that most of the invisible matter has to be non-luminous, non-baryonic and cold (i.e. non relativistic), therefore referred to as Cold Dark Matter (CDM) [1, 2, 3]. Moreover, both theory and observations point to the fact that CDM is constituted neither by baryons nor by neutrinos. The leading candidate as CDM particle is the so called Weakly Interacting Massive Particle (WIMP). A direct approach to WIMPs detection is to measure the nuclear recoils produced in the scattering of WIMPS off the nuclei of target materials in detectors placed deep underground. A review of the main technologies used in Dark Matter direct detection experiments is presented, with a special focus on experiments based on cryogenic noble liquids as target and detectors.
\end{abstract}

European Physical Society Europhysics Conference on High Energy Physics, EPS-HEP 2009, July 16 - 222009

Krakow, Poland

* Speaker. 


\section{Introduction}

The most important contribution to the matter content of the Universe is in Dark Matter, whose nature is still unknown. One can speculate about its features combining observations on all astronomical scales with the principles of the Standard Cosmological Model. From Big Bang nucleosynthesis we know that the majority of the missing mass in the Universe is non-baryonic [4]. In the framework of the Big Bang theory, it is natural to assume that during the early moments of the Universe, the Dark Matter particles were in thermal equilibrium with the others. However, in order to provide the present significant abundance and to satisfy the cosmological requirements $\Omega_{M} \cong 0.3$, they had to have decoupled before the present time [5]. This means that these particles represent a relic from the Big Bang, and their abundance is $\Omega_{\chi} h^{2}=\frac{m_{\chi} n_{\chi}}{\rho_{c}} \simeq \frac{3 \cdot 10^{-27} \mathrm{~cm}^{3} \mathrm{~s}^{-1}}{\left\langle\sigma_{a} v\right\rangle}$, where $m_{\chi}$ is the mass of the Dark Matter particles, $n_{\chi}$ is the present number density and $\left\langle\sigma_{a} v\right\rangle$ is the thermal average of the product of annihilation cross-section $\sigma_{a}$ and the velocity $v$. Therefore one obtains a value for $\left\langle\sigma_{a} v>\sim 10^{-26} \div 10^{-25} \mathrm{~cm}^{3} \mathrm{~s}^{-1}\right.$, very close to a typical weak interaction cross section, which is of the order of $\left\langle\sigma_{a} v\right\rangle \sim 10^{-25} \mathrm{~cm}^{3} \mathrm{~s}^{-1}$. Moreover, anisotropies in the Cosmic Microwave Background radiation (CMB), created by the fluctuations in the baryonic matter density, are not enough to create the distribution of matter of the present Universe and hot Dark Matter would not be able to assemble into the large scale structure we see today. The only solution to this puzzle is that Dark Matter particles have to be cold i.e. non-relativistic. Finally, considering the present conditions of the Universe, the Dark Matter particles have to be stable or, at least, have an average lifetime longer than the age of the Universe.

According to this picture, Dark Matter provides the first empirical evidence for particles beyond the Standard Model of particle physics. On the other hand, particles with the characteristics of the generic Weakly Interacting Massive Particles or WIMPs, are predicted in many supersymmetric extensions of the Standard Model, with largely different mass and interaction cross-section. An example and a favorite WIMP candidate is the neutralino, the lightest supersymmetric particle.

\section{Dark Matter direct detection}

If WIMPs exist, they are also the dominant mass in our own Milky Way [6], and, though they only very rarely interact with conventional matter, should nonetheless be detectable by sufficiently sensitive detectors on Earth. The WIMP flux on Earth should be of the order of $10^{5} \mathrm{~cm}^{-2} \mathrm{~s}^{-1}$, large enough to allow the detection of the nuclear recoils caused by their elastic scattering off target nuclei of Earth based detectors [7]. Thus, direct Dark Matter search experiments aim to detect the interactions of WIMPs in dedicated low background detectors, by measuring the rate, $R$, and energy, $E_{R}$, of the induced nuclear recoils and possibly, in directional experiments, the direction. Since the WIMP-nucleon relative velocity $v$ is non-relativistic, the recoil energy $E_{R}$ can be easily expressed in terms of the scattering angle in the center of mass frame, $\theta$ :

$$
E_{R}=\frac{|\vec{q}|^{2}}{2 m_{N}}=\frac{\mu_{\chi-N}^{2} v^{2}}{m_{N}}(1-\cos \theta)
$$

where $m_{N}$ and $m_{\chi}$ are the masses of the target nucleus and of the WIMP, $|\vec{q}|=\sqrt{2 m_{N} E_{R}}$ is the momentum transfer and $\mu_{\chi-N}=\frac{m_{\chi} m_{N}}{m_{\chi}+m_{N}}$ is the WIMP-nucleus reduced mass. The differential nuclear 


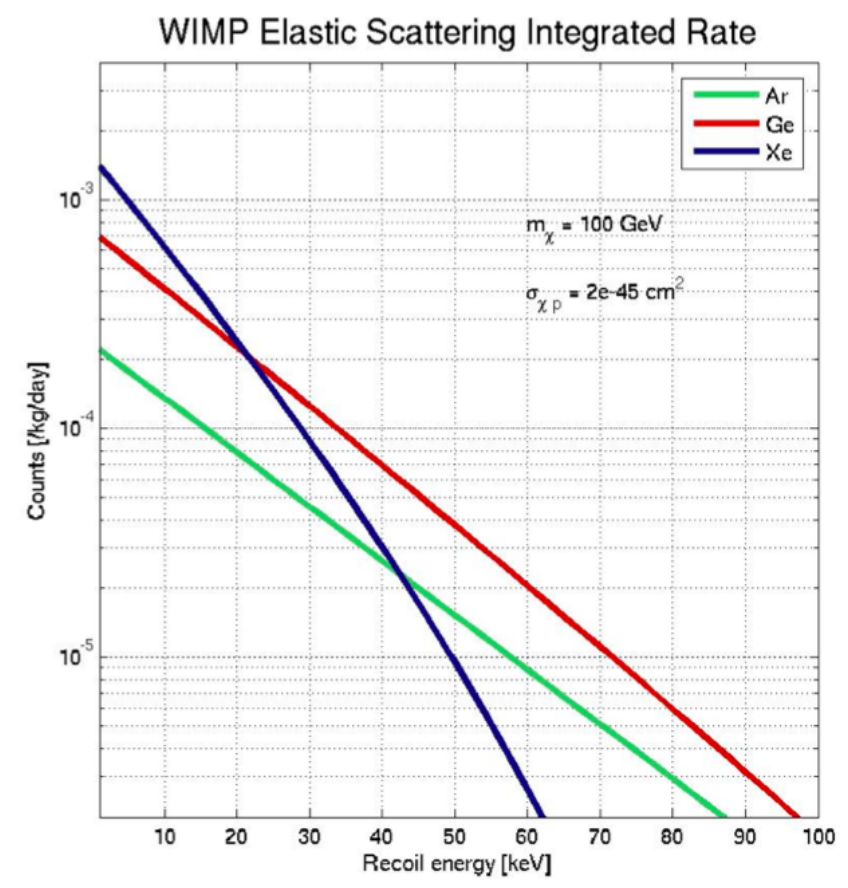

Figure 1: Total event rate (dashed curves) for WIMP elastic scattering off xenon, germanium and argon targets, calculated for a WIMP mass of $100 \mathrm{GeV} / \mathrm{c}^{2}$ and a WIMP-nucleus cross section of $10^{-45} \mathrm{~cm}^{2}$. As the atomic mass number increases, the rate increases at low energies, due to the coherence $\left(\sigma \propto \mathrm{A}^{2}\right)$, while as the recoil energy increases (i.e. the de Broglie wavelength approaches the nuclear dimensions) the rate decreases, due to the form factor. The influence of the form factor is more pronounced for high mass number elements. A low energy threshold is therefore mandatory for detectors with high mass number targets.

recoil rate induced by the WIMPs can be written as follows:

$$
R=\int_{E_{t h}}^{\infty} d E_{R} \frac{\rho_{0} \sigma_{0}}{m_{N} m_{\chi}} F^{2}\left(E_{R}\right) \int_{v_{\min }}^{v_{e s c}} v f(v) d v
$$

Here $E_{t h}$ is the energy threshold of the detector, $\rho_{0}$ is the local dark matter density, $\sigma_{0}$ is the cross section at zero momentum transfer, $f(v)$ is the WIMP velocity distribution in the galactic halo, $v_{m} i n=$ is the minimum velocity required for the WIMP to generate the recoil energy $E_{R}$ and $v_{e s c}$ is the galactic escape velocity. $F^{2}\left(E_{R}\right)$ is the nuclear form factor, which accounts for the fact that the de Broglie wavelength associated with the momentum transfer is of the same order as the nuclear dimensions; thus the bigger the nucleus the stronger this effect becomes.

The main astrophysical uncertainties lie in the velocity distribution $f(v)$ and in the dark matter density in the Earth vicinity, $\rho_{0}$. A common assumption is to use a Maxwellian distribution for $f(v)$ and a value of $0.3 \mathrm{GeVc}^{-2} \mathrm{~cm}^{-3}$ for $\rho_{0}$. A possible WIMP direct detection with directional experiments would provide a viable solution to the velocity distribution function problem.

If WIMPs are neutralinos, i.e. Majorana fermions, they can have only scalar or axial coupling with quarks, which, in this specific non-relativistic regime, translates into a spin-independent coupling and a coupling between the neutralino spin and the nucleon spin. In the spin-independent case, the full coherence results in a cross section $\sigma_{0} \propto A^{2}$, for a target nucleus of mass number $A$, while in the spin-dependent case the cross section is dominated by the total net spin of the nucleus. In most cases, the coherent term will dominate because it has the $\mathrm{A}^{2}$ enhancement. However, neutralinos with dominantly gaugino or higgsino states may only couple through the spin-dependent term.

As a result of the Earth motion relative to the WIMP halo, the event rate is expected to modulate with a period of one year with the maximum on the 2 nd of June. To detect this characteristic 
modulation signature, large masses are required, since the effect is of the order of $\sim 2 \%$ with respect to the total event rate.

A stronger diurnal direction modulation of the WIMP signal is also expected. The Earth rotation about its axis, oriented at angle with respect to the WIMP "wind", changes the signal direction by 90 degrees every 12 hours, with a resulting $30 \%$ modulation on respect to the total rate.

Nuclear recoils induced by WIMPs are detected exploiting the three basic phenomena associated with the energy loss of charged particles in target media: scintillation, ionization and heat. All the detectors used to perform this rare event search are also sensitive to the environmental radiation associated with cosmic rays and radioactivity in construction materials and the environment. At the current limits $[8,9]$ the expected WIMP rate is less than 0.1 event per day per kg and significant SUSY parameter space exists down to $10^{-6}$ event per day per $\mathrm{kg}$. Exploring this parameter space requires ton-scale detectors with nearly vanishing backgrounds.

Dark matter search experiments are located in deep-underground sites, to attenuate the cosmic muons flux by a factor $10^{5}$ to $10^{7}$. In addition, the detectors are typically enclosed by thick layers of absorbing materials (lead for $\gamma$ 's and hydrogen-rich compounds for neutrons), in order to reduce also the contribution due to the environmental radioactivity. A key requirement, and a challenge for all these experiments, is in the screening and selection of shielding and detector components, to allow significant background reduction. Detectors which the identification of multiple interactions in the target volume provides a powerful rejection method. Indeed, while the mean free path of a high energy $\gamma$-ray or a neutron is of the order of centimeters, the mean free path of a WIMP is of the order of a light-year, given its extremely low coupling with ordinary matter. In this way, it is possible to reject those events that have more than one interaction in the detector by defining a minimal time window between two consecutive events or packing the sensible volume within an active veto. However an efficient identification method of the events (generally based on threedimensional position reconstruction of the event's signature in the detector) is necessary in order to achieve a good rejection power. Finally in many dark matter direct search experiments background discrimination mechanisms are used, based on the fact that nuclear recoils (induced by WIMPs) and electron recoils (induced by the background particles) generate different signals in the detectors, due to the different nature of the particles.

\section{Dark Matter direct detection experiments (a selection)}

A large number of experiments aiming at a direct detection of Dark Matter are deployed in underground laboratories all around the world. Several are in construction phase. Only a small selection of them is presented in this review.

\subsection{The DAMA/LIBRA Experiment}

The DAMA/LIBRA experiment is presented as it is the only experiment which has to-date claimed a positive detection of Dark Matter WIMPs [10]. The project was designed in the1990 by an Italian group and installed at Gran Sasso underground laboratory. The detector (DAMA) was initially based on nine $9.7 \mathrm{~kg}$ highly radio-pure $\mathrm{NaI}(\mathrm{Tl})$ scintillators shielded from radioactive background. The collaboration has then upgraded the detector to a sensitive mass of $250 \mathrm{~kg}$ of 
$\mathrm{NaI}(\mathrm{Tl})$. This new experiment, called LIBRA, is running since March 2003. The threshold provided for both experiments is $2 \mathrm{keV}$.

The DAMA experiment belongs to the first generation of dark matter direct detection experiments, which require a large detector exposure. Although the $\mathrm{NaI}(\mathrm{Tl})$ scintillator provides some discrimination between nuclear recoils and electron recoils based on pulse shape, the collaboration published its data without any background reduction. Using a seven-year exposure ( 0.29 ton $\times$ year), the DAMA collaboration reported a $6.3 \sigma$ C.L annual modulation signal in WIMP-proton elastic scattering. Recently, the LIBRA experiment has confirmed the observation with an exposure of 0.53 ton $\times$ year.

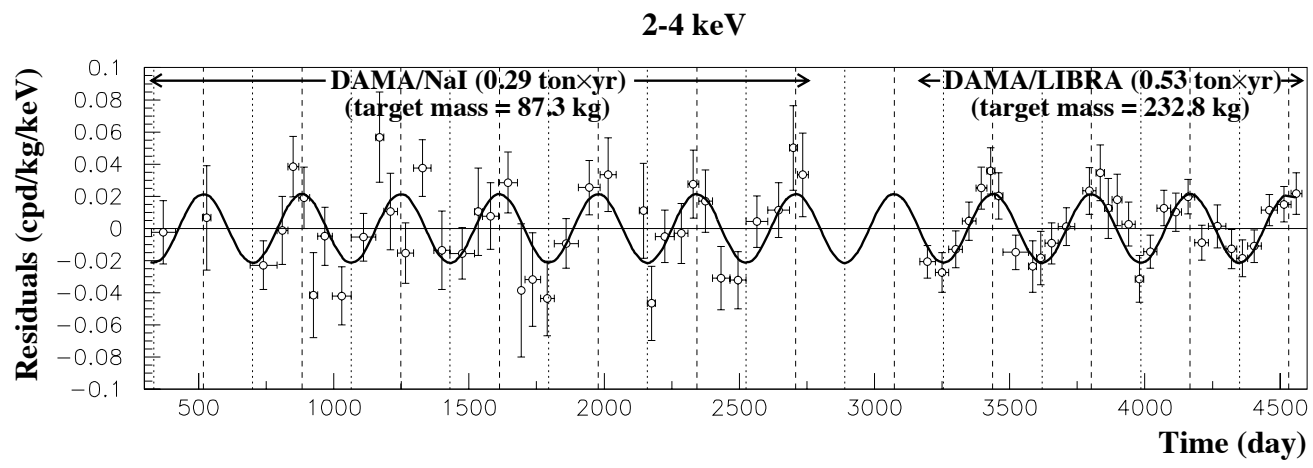

Figure 2: DAMA annual modulation signal from a model independent fit to the cosine function, showing a period of oscillation of $1.00 \pm 0.01$ year and offset $t_{0}$ equal to $140 \pm 22$ days.

The DAMA/LIBRA evidence for the annual modulation is clear but only in the lowest energy bins (2-6 keV corresponding to a nuclear recoil energy of 22-66 keV for interaction on Iodine nuclei, see Figure 2) where the understanding of the efficiencies is particularly important. The origin of this clear modulation and its interpretation continue to be widely disputed, although many studies have been performed by the collaboration regarding various possible systematic effects.

\subsection{The COUPP Experiment}

The COUPP (Chicagoland Observatory for Underground Particle Physics) experiment exploits the bubble chamber technology. By tuning the superheated liquid in such a way that only particles with $d E / d x>50 \mathrm{keV} / \mu \mathrm{m}$ (like scattered nuclei) can create a bubble nucleation, the detector is extremely insensitive to electron recoil events $\left(>10^{10}\right.$ rejection power). All other background particles (like muons, $\gamma$-rays, X-rays and $\beta$ s) are well below the nucleation threshold. The energy threshold for recoiling nuclei is as low as $\sim 5 \mathrm{keV}$. Precautions are being taken in order to reduce the inhomogeneous bubble nucleation by mildly superheating the liquid. A notable source of background in the COUPP detector are the $\alpha$ particles from natural occurring radioactive isotopes, mainly from ${ }^{222} \mathrm{Rn}$ and its daughters.

A first prototype with $1.5 \mathrm{~kg}$ of $\mathrm{CF}_{3} \mathrm{I}$ was operated at the Fermilab NuMI underground tunnel ( $\sim 300$ meters water equivalent). Few examples of different type of events detected by this prototype are shown in Figure 3 left. Such small detector provided the most stringent limit on 
pure proton spin-dependent interaction for low mass WIMPs [11], excluding the low mass region favored by a spin-dependent interpretation of the DAMA signal (see Figure 3 (right)).

A $60 \mathrm{~kg} \mathrm{CF}_{3}$ I COUPP detector has been constructed, and will likely operate at SNOLAB.
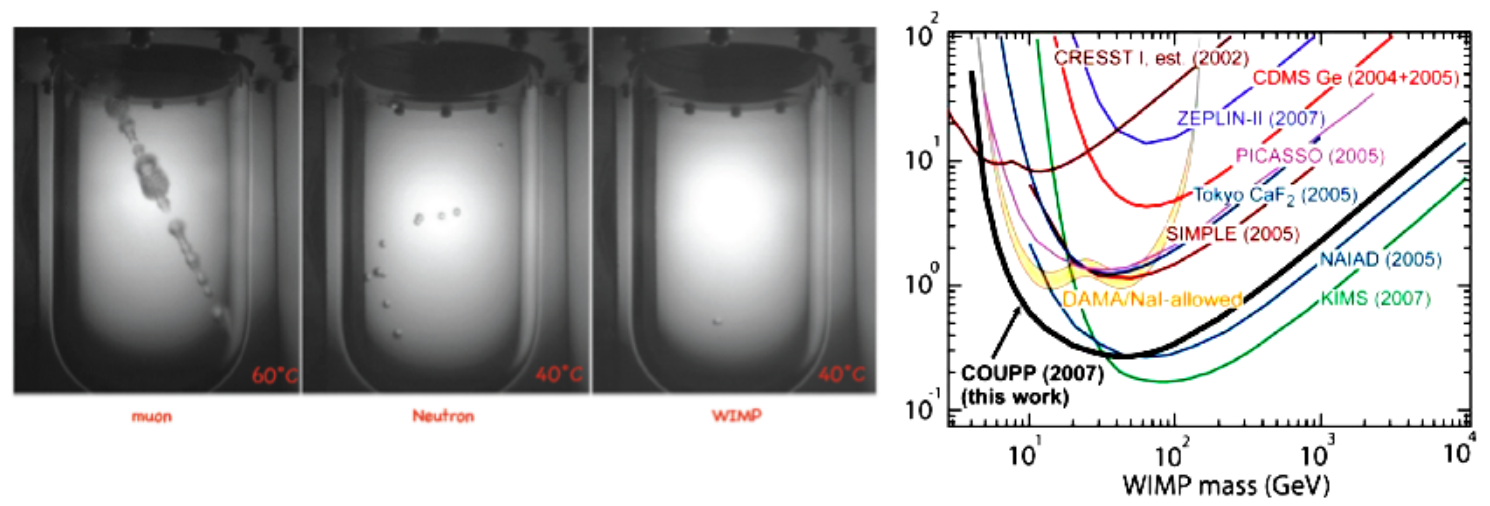

Figure 3: (left) examples of different type of particles detected at different temperature and pressure conditions in the $1.5 \mathrm{~kg}$ COUPP prototype operated at the Fermilab NuMI underground tunnel. (right) Limit on the spin-dependent (pure) proton-WIMP interaction cross section versus WIMP mass, obtained with the COUPP prototype.

\subsection{The XENON Experiment}

Of the several direct detection experiments based cryogenic noble liquids, currently only two are operating underground: XENON using liquid Xe and WARP using liquid Ar, both with a target mass on the $100 \mathrm{~kg}$ scale. These experiments exploit the time projection chamber (TPC) technology, with simultaneous detection of the ionization (via proportional scintillation) and the direct scintillation signals. The amplitude and timing of the signals, as well as the 3-dimensional event localization capability, enables these TPCs to effectively identify and reject background. The XENON collaboration is following a phased approach to the direct detection of WIMPs in liquid $\mathrm{Xe}$, with a series of detectors of increasingly larger mass and lower background. The goal is to realize within the middle of next decade an experiment with a ton scale fiducial target (XENON1T) to search for WIMPs with almost four orders of magnitude better sensitivity than the current best limit. After the successful results of the first $10 \mathrm{~kg}$ scale prototype, XENON10 [9, 12], the XENON collaboration has designed and built a second generation experiment with a mass increase of a factor 10 and a background reduction of a factor 100, in order to achieve the sensitivity goal of $\sim 2 \times 10^{-45} \mathrm{~cm}^{2}$ for the WIMP-nucleus spin-independent cross section at a WIMP mass of $\sim 30$ $\mathrm{GeV} / \mathrm{c}^{2}$, as shown in Figure 4.

A schematic view of the XENON100 detector is shown in Figure 6. The inner TPC target is surrounded by an active liquid xenon veto. Both target and veto are contained in a doublewalled vacuum cryostat made of low activity stainless steel (SS). The total mass of Xe required to fill the detector is $170 \mathrm{~kg}$, of which approximately $65 \mathrm{~kg}$ are in the fiducial volume (target). The light readout is based on a total number of 242, 1 inch square photomultiplier tubes (PMTs) (Hamamatsu R8520-06-AL), with selected low radioactivity materials; seventy of such PMTs have higher quantum efficiency, better than $30 \%$. The target is seen by a top and a bottom array of 


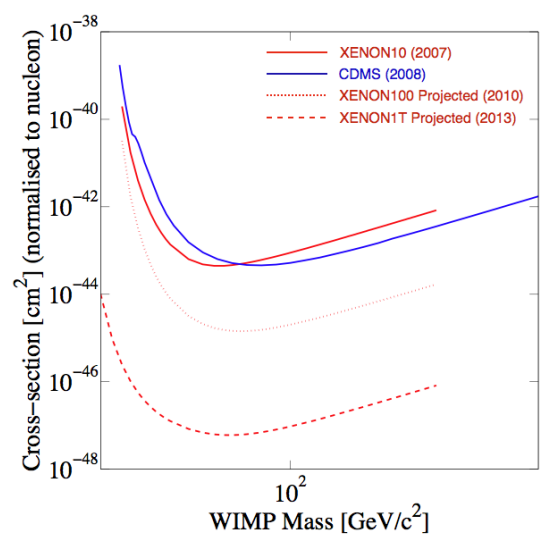

Figure 4: WIMP-nucleon cross-section upper limit (90 \% C.L.) from direct dark matter search experiments. The projected upper limits for all the XENON phases are shown as dashed lines.

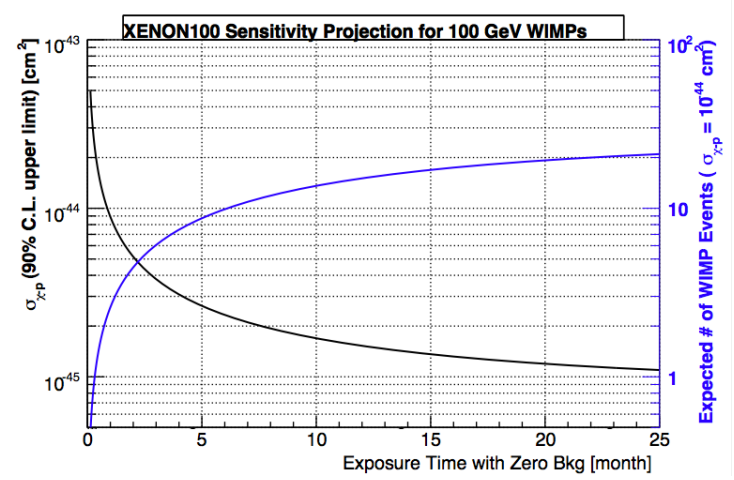

Figure 5: XENON100 sensitivity as function of the exposure.

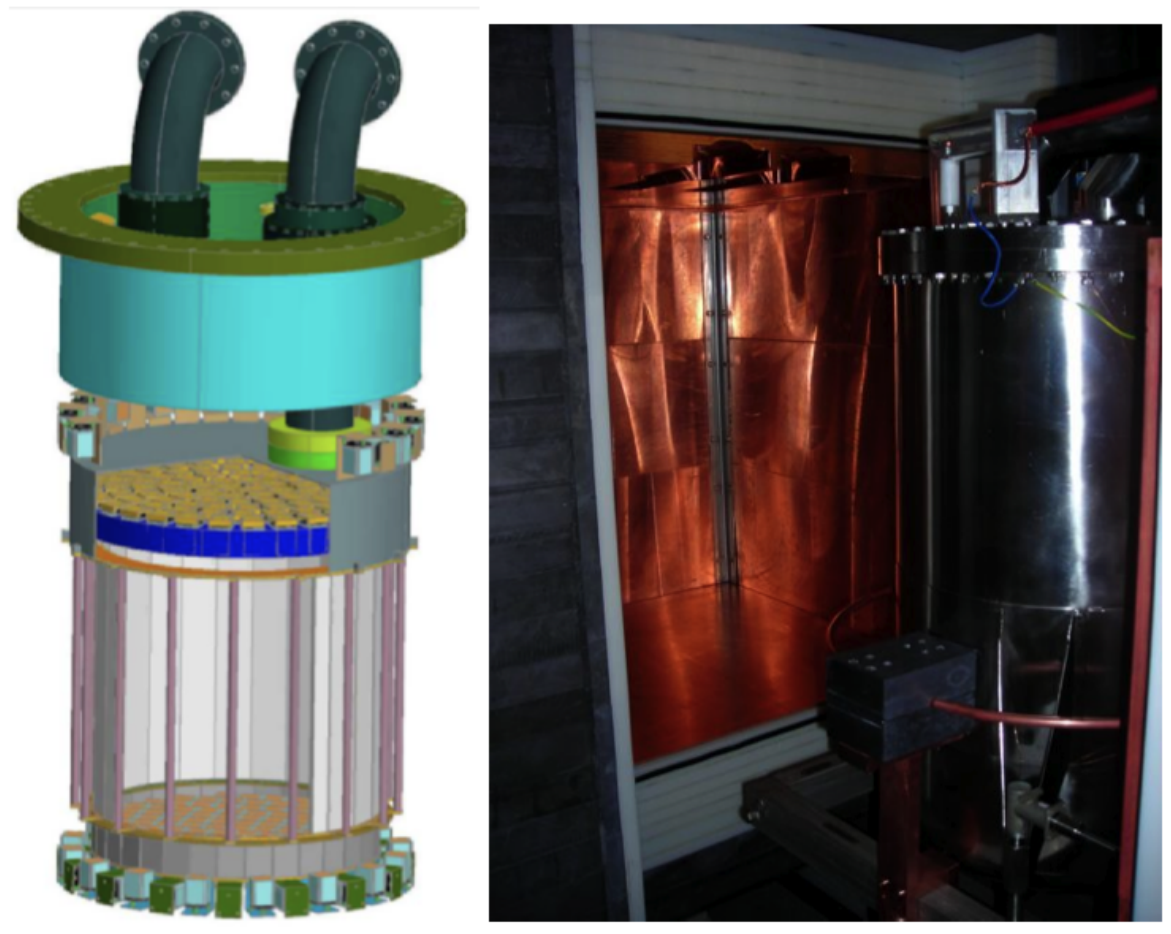

Figure 6: Schematic view of the XENON100 detector design.

PMTs, as in XENON10. An important background rejection feature of the XENON100 detector is the ability to localize events in 3 -D with a spatial resolution a few $\mathrm{mm}$ in $\mathrm{x}-\mathrm{y}$ plane and $<1 \mathrm{~mm}$ 
in $\mathrm{z}$.

The target is enclosed by a teflon structure, made with interlocking panels. Teflon is used as an effective UV light reflector and as an electrical insulator. The TPC is defined by four wire meshes, two in the liquid and two in the gas. The bottom liquid mesh acts as cathode and is connected to the top liquid one through 30 field shaping rings, placed at a mutual distance of $1 \mathrm{~cm}$. The top two meshes, together with the one below the liquid level, define the gas proportional scintillation region. The wire meshes and top PMT array are mounted in a SS cylinder closed on top by a "diving bell", keeping the liquid level at a precise height. A positive pressure in the bell is provided by the gas returning from the gas recirculation system, which continuously purify the Xe through a high temperature getter.

For a TPC like XENON100, where the maximum drift length that ionization electrons have to travel is $30 \mathrm{~cm}$, an essential requirement is the reduction of any electronegative impurity at very low levels. In the XENON10 detector an electron lifetime of $1.8 \pm 0.4 \mathrm{~ms}$ was achieved. Although more challenging, similar levels are being achieved in XENON100.

To reduce the radioactivity from the detector components and shield materials, a careful selection of all the materials has been done. This was performed based on the results of the radioactivity screening of many samples, mainly performed with High Purity Germanium spectrometers. Similarly important in terms of radioactivity contamination, is the requirement for very low level contamination of radioactive ${ }^{85} \mathrm{Kr}$ in Xe. A cryogenic distillation column has been tested and operated on XENON100 to reduce the ${ }^{85} \mathrm{Kr}$ concentration below the $50 \mathrm{ppt}$ (part per trillion) required by the sensitivity goal.

The commissioning of the XENON100 experiment is underway and calibration and first detector characterization have started. The first scientific run is expected to start at the beginning of 2010.

\subsection{The WArP Experiment}

The WArP experiment is also based on a double-phase TPC technology. In this case the sensitive medium consists of $100 l$ liquid argon [13]. It is a new generation Dark Matter direct detection experiment, whose technology has been successfully tested with a $2.3 l$ prototype [14].

In addition to the background discrimination accomplished by the simultaneous detection of primary and secondary scintillation signal, a suitable pulse shape discrimination of the primary scintillation signal can be used, given the wide separation in rise time between the fast (5 ns) and the slow $(\sim 1.4 \mu \mathrm{s})$ components. In addition, and like the XENON100 experiment, WArP relies on the power of the 3-D event position reconstruction for additional background rejection. The experiment is located at the Gran Sasso Laboratory. A first calibration run was carried out in May 2009.

The WArP $100 l$ detector layout is schematically shown in Figure 7. It consists of an external passive shield (a polyethylene $70 \mathrm{~cm}$ thick layer as $\mathrm{n}$-shield and a lead $10 \mathrm{~cm}$ thick gamma-shield), a 15 ton LAr cryostat (a double wall cryogenic vessel insulated with vacuum and super-insulation and made of stainless steel selected for low radioactive contamination) containing the inner detector (100 $l$ of active LAr target), the active veto formed by about 8 ton of LAr around the inner detector and, externally to the active veto, also immersed in LAr, a $10 \mathrm{~cm}$ thick shield of polyethylene. 


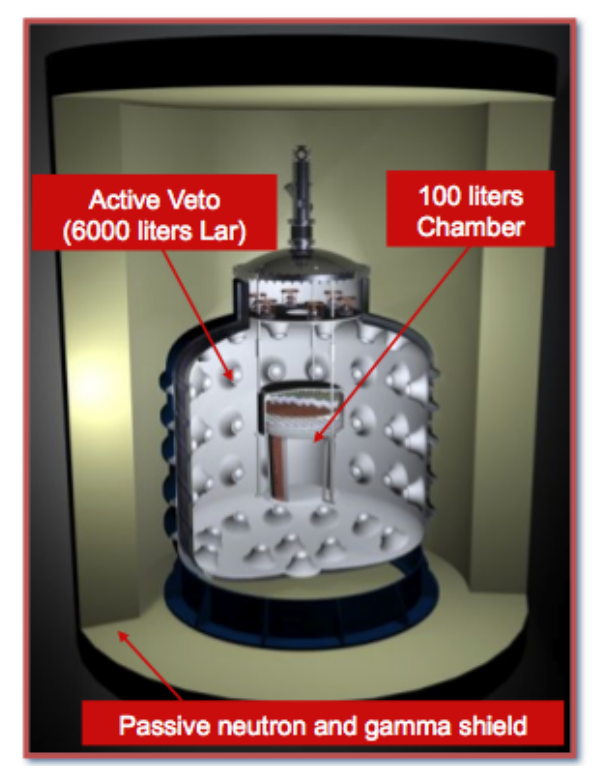

Figure 7: Ionization yield versus recoil energy for all the events passing the analysis cuts as published in [8].

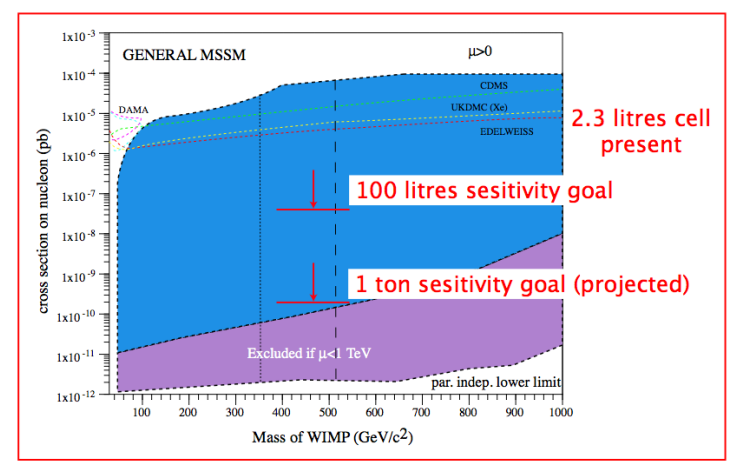

Figure 8: Spin-independent WIMP-nucleon crosssection upper limits (90 \% C.L.) versus WIMP mass, set by the WArP $2.3 l$ chamber and projected sensitivity for the 1001 and 1 ton scale detectors.

The inner detector, equipped with field-shaping electrodes for the drift field, grids for extraction of ionization electrons from liquid to the gas phase and proportional light production, and PMTs for the readout of the light signals, is suspended at the center of the LAr veto volume. This volume works as active shield (veto) against gamma and neutron backgrounds. The minimum thickness of the active LAr shield is $60 \mathrm{~cm}$. It's viewed by photomultipliers that are held in place by PEEK supports that are connected to a thin copper structure that delimits the external surface of the active shield volume. On the $\mathrm{Cu}$ structure are also mounted the basic elements of the wavelength shifting/reflecting layer (TetraPhenylButadiene wavelength shifter, deposited on a highly reflective plastic substrate). A total of 4363 " phototubes are installed in the active shield for $10 \%$ photocathode coverage of the inner surface and a nominal threshold of $10 \mathrm{keV}$ for argon recoils (10 photoelectrons). The inner detector and the active shield are optically separated (to avoid vetoing of events occurring in the central volume).

The cosmogenic beta decaying ${ }^{39} \mathrm{Ar}(565 \mathrm{keV}$ endpoint, and half life-time of $260 \mathrm{yr}$ ) in natural argon is unavoidable and is the limiting factor for the WArP experiment for WIMP direct detection. The specific activity of ${ }^{39} \mathrm{Ar}$ in natural argon is $1 \mathrm{~Bq} / \mathrm{kg}$. Isotopic separation by centrifugation or differential thermal diffusion are established techniques for separation of ${ }^{39} \mathrm{Ar}$ from ${ }^{40} \mathrm{Ar}$, but on a multi-ton scale could become extremely expensive and require a long production time. Argon from natural gas wells is of potential interest because ${ }^{39} \mathrm{Ar}$ production induced by cosmic rays is strongly suppressed underground. 


\subsection{The CDMS experiment}

To detect WIMPs interactions, CDMS uses the so called ZIP (Z-dependent Ionization Phonon) detector technology, consisting in disc-shaped germanium $(250 \mathrm{~g})$ or silicon (100 g) crystals. The final CDMS-II experiment consists of a total of 30 detectors, 19 Germanium and 11 Silicon crystals. The technique allows the simultaneous detection of ionization and phonon signals. One face of the disc is covered by a thin layer of aluminum and 1024 tungsten transition-edge sensors (TES) which are evenly distributed over the surface. The Al layer provides the phonon-phonon coupling between the two materials, so that the athermal phonons can pass the interface depositing their energy into the Al by breaking Cooper pairs. These pairs are tunneled into the TES's at the superconducting transition temperature, and thus a small variation in the TES temperature will cause a significant change in the TES resistance ( $\sim 10 \mathrm{~m} \Omega$ ), which is then read out. The other face of the detector, covered by $A l$, allows the collection of the charges using an electric field. The double detection technique provides both a high sensitivity and an efficient discrimination method.

Electron recoil events from surface contamination result in a reduced ionization signal, leading to the leakage of such events into the nuclear recoil identification region. A phonon timing cut, developed by the collaboration, provides an effective discrimination against these events and the resulting rejection power for the electron recoil background is of the order of $10^{6}$.

The data acquired between October 2006 and July 2007 with the complete detector have been analyzed and the results published in 2008 for a total exposure of $121.3 \mathrm{~kg}$-day [8]. Figure 9 shows the event distribution in the ionization yield - recoil energy parameter space, which defines the acceptance regions for the electron recoil background and the nuclear recoil interesting signals. The upper limit (at $90 \%$ C.L.) on the spin-independent WIMP-nucleus cross section reported by CDMS is shown in Figure 10, with a minimum of $6.6 \times 10^{-44} \mathrm{~cm}^{2}$ for a WIMP mass of $60 \mathrm{GeV} / \mathrm{c}^{2}$.

\subsection{Directional experiments}

Due to the Sun's motion around the galactic center, and its relative motion in the WIMP halo, WIMPs coming towards the Earth have to have specific directions. Knowing that the WIMP wind comes from the Cygnus constellation direction, a strong forward/backward asymmetry in the direction of possible WIMP signals is expected, as shown in Figure 11. The background, being uniformly distributed around the detector and having a characteristic isotropic directionality, cannot mimic such WIMP directional signal, and a total of 10 events is sufficient to claim a positive detection result.

Gas based TPCs, where a recoil event with a few keV has detectable size trajectory in the gas medium, can provide such directional information. The fact that electron recoils have smaller stopping power than that of nuclear recoils allows a viable background rejection method, by measuring both the range and the ionization. A challenge for this type of Dark Matter direct detection experiments is the three-dimensional event reconstruction.

There are several directional experiments under construction or already operating all around the world, using different type of gas and different technologies for signal read-out:

- The DRIFT-II experiment at the Bulby mine is a $1 \mathrm{~m}^{3}$ multi wire TPC filled with 40 torr of $\mathrm{CS}_{2}(167 \mathrm{~g})[15]$. 

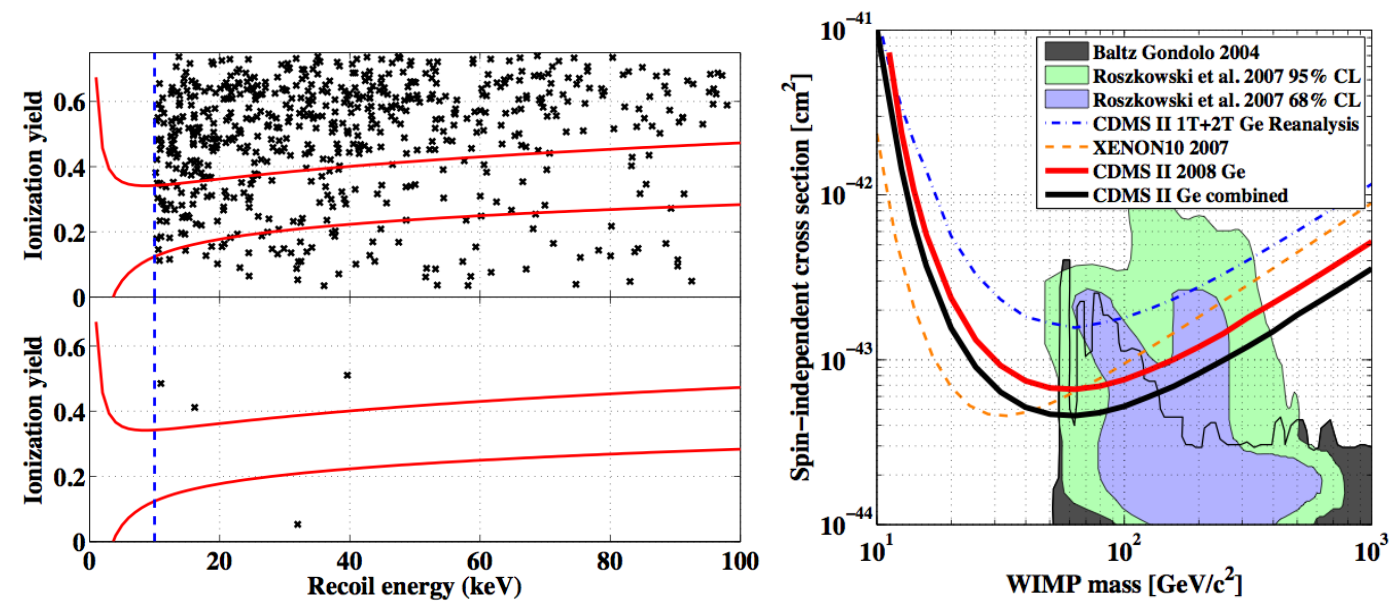

Figure 9: Ionization yield versus recoil energy for all the events passing the analysis cuts as published in [8].

Figure 10: Spin-independent WIMP-nucleon cross-section upper limits (90 \% C.L.) versus WIMP mass, set by the CDMS-II experiment for a total of $121.3 \mathrm{~kg}$-day.

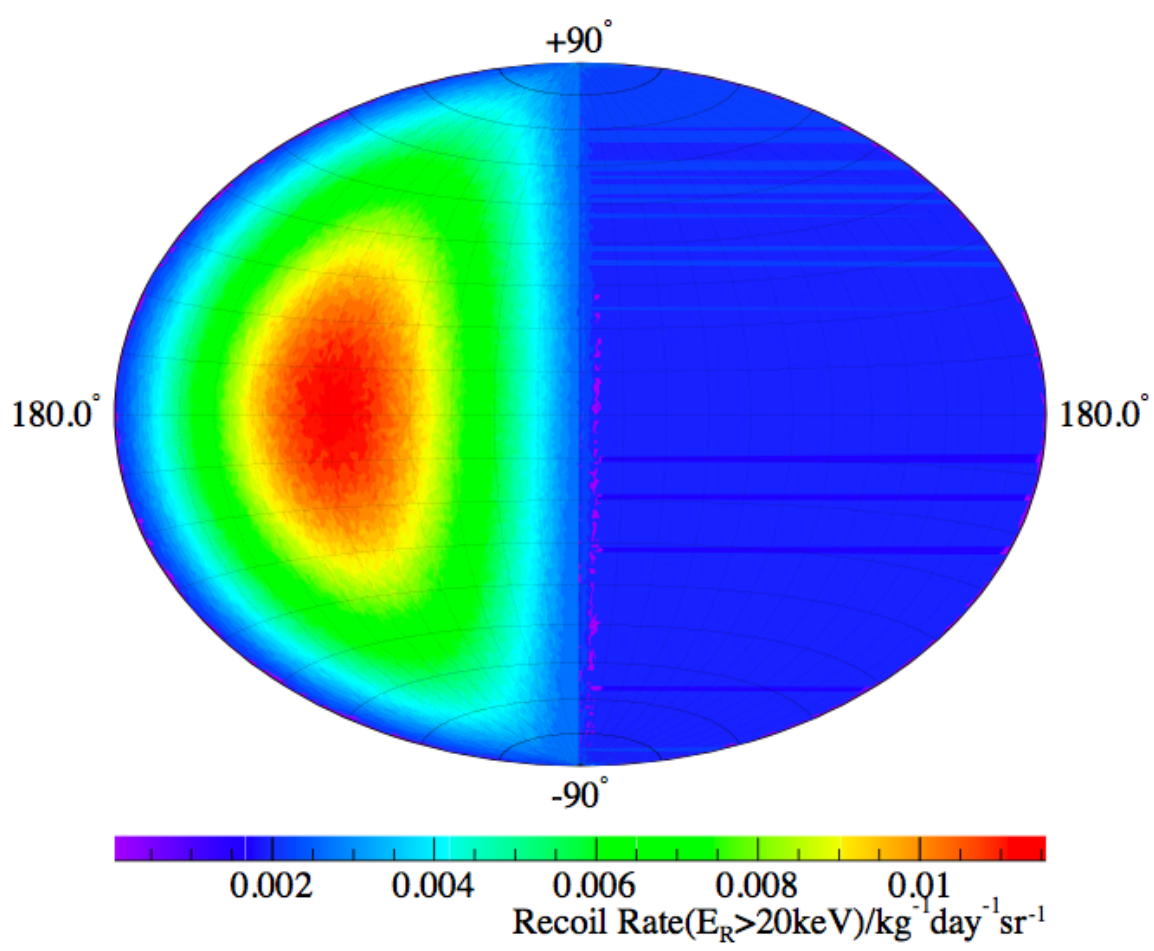

Figure 11: Example simulation of WIMP flux plotted in galactic coordinates for a Maxwell-Boltzmann halo with $v_{0}=220 \mathrm{~km} \mathrm{~s}^{-1}$. 
- The DM-TPC experiment at MIT consists of two $10^{-2} \mathrm{~m}^{3}$ TPC filled with 50 torr of $\mathrm{CF}_{4}$, where the three-dimensional readout is done with CCDs while the energy information is provided by photomultipliers [18].

- The NEWAGE experiment at the Kamioka mine is a $23 \times 28 \times 30 \mathrm{~cm}^{3}$ TPC filled with 150 torr of $\mathrm{CF}_{4}$ and equipped with microwell for energy and three-dimensional event reconstruction [16].

- Finally, the MIMAC experiment at Saclay consistsy of several TPC modules filled with ${ }^{3} \mathrm{He}$ and $\mathrm{CF}_{4}$, equipped with $3 \times 3 \mathrm{~cm}$ Micromegas with pixellized anode, suitable both for energy and event trajectory measurements [17].

\section{Summary}

The identity of Dark Matter particles still remains a mystery, but the potential for breakthrough in the coming decade is very likely. Direct detection experiments have made significant progress in recent years, driven in part by an aggressive competition worldwide. They complement indirect and collider searches. Several $100 \mathrm{~kg}$ scale experiments are presently in operation underground or under construction, promising interesting new results in the coming years. For XENON100, results are expected in 2010. If the cross-section is at the $10^{-8} \mathrm{pb}$ level, as in some favored SUSY models, the experiment should start seeing a handful of WIMP events and that is very exciting. Equally important is that for the first time a low background, massive target, other than NaI, can probe annual modulation.

Increasing mass while keep lowering backgrounds is the rule of the game and noble liquids continue to advance towards this goal. Ton scale experiments are technically feasible and will follow quickly the current generation experiments. A direct detection signal, from either or both SI and SD interactions, needs to be validated with more than one target and concept: the current zoo of experiments is therefore vital for this research field. Directional experiments are also advancing at good pace and will most probably provide the ultimate "smoking gun" for a DM signal.

\section{References}

[1] W. Freedman and M. Turner, Rev. Mod. Phys., 75, 1433 (2003).

[2] D. Clowe et al., ApJ, 648, L109 (2006).

[3] M. J. Jee et al., Astrophys. J. 661 (2007) 728.

[4] K. Griest, M. Kamionkowski, and M. S. Turner, Phys. Rev. D 41 (1990) 3565.

[5] M. S. Turner, E. W. Kolb, 1990, The early Universe (Frontiers in Physics), (Addisn-Wesley, U.S.).

[6] M. R. Merrifield, Astronom. J., 103 (1992) 1552.

[7] M. W. Goodman, E. Witten, Phys. Rev. D 31 (1985) 3059.

[8] Z. Ahmed et al. [CDMS Collaboration], Phys. Rev. Lett. 102 (2009) 011301 [arXiv:0802.3530 [astro-ph]].

[9] J. Angle et al. (XENON10 Collaboration), Phys. Rev. Lett. 100, 021303 (2008). 
[10] R. Bernabei et al. (DAMA Collaboration), Eur. Phys. J. C 56 (2008) 333

[11] E. Behnke et al., Science 319 (2008) 933;

[12] J. Angle et al. (XENON10 Collaboration), Phys. Rev. Lett. 101 (2008) 091301.

[13] R. Brunetti et al., WARP: WIMP Argon Programme, Proposal for WARP to INFN, March 2004, available at http://warp.pv.infn.it.

[14] R. Brunetti et al., New Astron. Rev. 49, 265 (2005).

[15] G. J. Alner et al. (DRIFT Collaboration), Nucl. Instr. Meth A 555 (2005) 173. S. Burgos et al. (DRIFT Collaboration), Astropart. Phys. 28 (2007), no. 4Đ5, 409.

[16] T. Tanimori et al., Phys. Lett. B 578 (2004) 241.

[17] D. Santos et al., J. Phys. Conf. Ser. 65 (2007) 012012.

[18] A. Roccaro et al., Nucl. Instrum. Meth. A 608 (2009) 305 [arXiv:0906.3910 [physics.ins-det]]. 